\title{
Patient Satisfaction after Total Hip Arthroplasty Comparing the Direct Anterior Approach with other Standard Approaches
}

\section{Joseph T Moskal' ${ }^{1}$ John A Scanelli² and Susan G Capps ${ }^{3 *}$}

${ }^{1}$ Carilion Clinic Orthopaedics, Virginia Tech Carilion School of Medicine, Roanoke, Virginia, USA

${ }^{2}$ University of Virginia, Department of Orthopedic Surgery, Charlottesville, Virginia, USA

${ }^{3}$ Bensol, Warsaw, Indiana, USA

\begin{abstract}
A telephone questionnaire was utilized to assess whether patients who underwent staged bilateral total hip replacement using the direct anterior approach for one hip replacement, and a different surgical approach for the other, could detect a difference in their post-operative recovery. When comparing patients who preferred the results of one hip replacement over another, the DAA was associated with more patient satisfaction, a quicker recovery, less pain, better function, and better fulfillment of pre-operative expectations, while there was no difference in hip strength between groups. These results suggest randomized controlled trials are worth pursuing to determine if a clinically meaningful difference exists between the direct anterior approach and other surgical approaches.
\end{abstract}

Keywords: Direct Anterior Approach (DAA); Primary total hip arthroplasty; Bilateral total hip arthroplasty; Patient satisfaction

\section{Introduction}

The direct anterior approach (DAA) for total hip arthroplasty has gained recent interest following the reported claims of a quicker recovery [1-3], minimal post-operative pain [4], and a low dislocation rate without hip precautions [5,6] compared to other surgical approaches. These outcome measures are mostly attributed to the internervous plane of the direct anterior approach that does not require splitting muscle to achieve exposure of the hip joint [7]. Physiologic markers of muscle damage [8], gross inspection of cadaveric specimens [9], and MRI evidence [10] all support the conclusion that the direct anterior approach is associated with less damage to muscle compared to other traditional approaches in total hip arthroplasty. The clinical significance of these claims and whether the DAA is directly responsible for improving the short or long-term outcomes requires further investigation.

The patient population undergoing total hip replacement includes younger and more active patients who desire to return to work and recreational activities soon after surgery without adversely affecting the short- or long-term outcome. Whether or not one surgical approach is better able to achieve this over another has not been proven. Patient satisfaction is an important outcome measure of total hip arthroplasty success $[11,12]$; therefore we searched the available literature and found no articles that directly assess patient satisfaction or patient perception of post-operative recovery with the direct anterior approach versus other surgical approaches. We hypothesized that patients would perceive no difference between the direct anterior approach and alternative surgical exposures for total hip arthroplasty with respect to the post-operative duration of recovery, pain, or more closely meet pre-operative expectations.

\section{Materials and Methods}

The clinical database of the senior author was searched for patients with bilateral total hip arthroplasty who had one hip replaced with the direct anterior approach and the other with a different approach (anterolateral, modified direct lateral, and posterior). Primary total hip arthroplasty was the index procedure for all hips. Any patient who had revision surgery or a prior history of hip surgery before primary total hip arthroplasty was excluded from the study group. This resulted in a study group of 70 patients who were identified and then contacted following IRB approval. The majority of patients had their non-direct anterior approach total hip arthroplasty performed by the senior author; the remainder were patients who moved into the senior author's practice area after their first total hip arthroplasty. Each patient received a letter explaining that participation in the study was voluntary and their choice to participate would not affect their postoperative care or follow-up in any way.

A telephone questionnaire was developed to assess patient perception of post-operative pain, length of recovery, whether preoperative expectations were better met with one hip replacement compared to the other, and overall satisfaction after each total hip replacement. Questions were also designed to assess patient perception of different short-term and long-term outcome measures. Responses to all questions were in the form of "left" or "right" hip replacement to minimize the risk of introducing bias for one approach over another. Patients were also given the option to select "no difference" if they felt both hip replacements performed the same with respect to the outcome measure tested.

A power analysis was performed and established that between 60 and 70 patients was necessary to obtain sufficient power to detect a clinically significant difference in patient responses to the questionnaire. A research assistant contacted 70 patients and administered the telephone questionnaire. Suitable responses were obtained from 69 patients, of these four had undergone revision total hip arthroplasty and thus their responses were unusable, therefore the final population was 65 patients.

To account for the effects of multi-modal pain management, we performed an additional subgroup analysis comparing the responses of patients who had multi-modal pain management only with the

*Corresponding author: Susan G Capps, BENSOL, 488 East Bell Drive Warsaw, Indiana 46582, USA, Tel: (574) 453-1272; Fax: (574) 269-6572; E-mail:writing@sgcappsphd.com

Received August 26, 2013; Accepted December 28, 2013; Published February 10,2014

Citation: Moskal JT, Scanelli JA, Capps SG (2014) Patient Satisfaction after Total Hip Arthroplasty Comparing the Direct Anterior Approach with other Standard Approaches. Surgery Curr Res 4: 174. doi:10.4172/2161-1076.1000174

Copyright: ( 2014 Moskal JT, et al. This is an open-access article distributed under the terms of the Creative Commons Attribution License, which permits unrestricted use, distribution, and reproduction in any medium, provided the original author and source are credited. 
direct anterior approach operation to those who had it for both hip replacements. The senior author began to routinely use multi-modal pain management in 2003, and this date was used to stratify the two sub-groups of patients. We also performed an additional subgroup analysis by stratifying patients according to age greater than or less than 70, and gender to see if either sub-group had a preference for one hip replacement over another.

Statistical analysis of resulting data was performed using JMP 10.0 .0 statistical analysis software (SAS Institute Inc., Cary, North Carolina). Given the nature of the response data (DAA-side versus non-DAAside, or possible No-Difference), contingency analyses were employed.

\section{Results}

The study group consisted of 65 patients, 29 males (44.62\%) and 36 females $(55.38 \%$ ), with a mean age of 69.01 years (SD 12.94, range 30 to 90 years). Each patient only had one operation on each hip (primary total hip replacement) with a pre-operative diagnosis of degenerative joint disease in 54 patients, avascular necrosis in 10 patients and rheumatoid arthritis in 1 patient. The average time between hip replacements was 79.49 months (SD 55.41, range 2 to 294 months). The mean BMI was $29.40 \mathrm{~kg} / \mathrm{m}^{2}$ (range 20 to $53 \mathrm{~kg} / \mathrm{m}^{2}$ ). The surgical approaches utilized to perform the contralateral hip replacement included 4 anterolateral, 38 modified direct lateral, and 23 posterior.

When comparing patients who preferred the results of one hip replacement over another, the DAA was associated with a quicker recovery $(\mathrm{P}<0.0001)$, less pain during the acute hospitalization $(\mathrm{P}<0.0001)$, easier time putting on socks and shoes $(\mathrm{P}<0.0001)$, and more fulfillment of pre-operative expectations $(\mathrm{P}<0.0001)$, while there was no difference in hip strength $(\mathrm{p}=0.89$ ) or duration of post-operative physical therapy $(\mathrm{p}=0.06)$ between groups.

$78 \%$ of patients (51 of 65) preferred the results of one hip replacement to the other. Of those patients who had a preference, $78 \%$ of those patients preferred the DAA (40 of 51). Patients less than 70 years of age were also more likely to prefer the results of their DAA hip replacement compared to older patients.

However, when age was further analyzed, patients less than 70 years of age reported more satisfaction with the post-operative function of their hip replaced through the DAA, and found their pre-operative expectations were more closely met after hip replacement through the DAA. They also perceived a faster surgical recovery, and were more likely to recommend a hip replacement to a friend or loved one based on the results of their DAA hip replacement.

$69 \%(n=18 / 26)$ of patients responded they were able to return to work sooner following the DAA operation compared to an alternative approach.

\section{Discussion}

Total hip arthroplasty has an excellent track record for providing durable pain relief, improving quality of life and restoring function for patients suffering from hip arthritis [13]. We designed a questionnaire to assess whether the direct anterior approach is capable of providing less post-operative pain, a more rapid surgical recovery, improved function, and could better meet the expectations of patients treated with total hip arthroplasty. The data from this questionnaire suggests that the majority of patients perceive less acute pain, a quicker recovery, and is more satisfied with their surgical experience with the direct anterior approach compared to other traditional approaches. However, patients were unable to appreciate a significant difference in their long-term function, strength or pain relief with the direct anterior approach. This finding is consistent with other studies comparing the direct anterior approach to other surgical approaches that show equivalent outcomes in the long term [2], and variable rates of improvement in the short term between 6 weeks to 12 months $[2,7,14,15]$.

We had intended to account for the effects of multi-modal pain management and patient age (subdivided at 70 years of age), however, due to the large number of responses to questions that were "not different" and null (no response given), these analyses were not possible.

There is conflicting evidence from clinical outcome studies comparing the direct anterior approach for total hip arthroplasty to other surgical approaches. Measures of post-operative gait, pain and length of hospitalization are frequently compared in these investigations.

Patients in our study group did not perceive a significant difference in strength or post-operative function with the DAA. These findings are mostly in agreement with other studies that assessed measures of gait performance after total hip arthroplasty comparing the DAA to other surgical approaches. Klausmeier et al. [15] found an improvement in gait velocity and peak flexor moments at 6 weeks in patients with the DAA, however most of the isometric and dynamic measures of gait function were similar between the DAA group and the anterolateral group at 6 weeks and 16 weeks. Maffiuletti et al. [16] found no difference in measures of gait performance between the DAA and the posterior approach at self-selected and fast walking speeds. WOMAC function scores did not differ either; however there was a higher reported incidence of WOMAC stiffness in the posterior group. Mayr et al. [17] found a larger improvement in cadence, stride time and length, as well as walking speed with the DAA group compared to an anterolateral group. The small number of patients in each study group from Mayr et al. [17] studies makes it difficult to draw any meaningful conclusions.

However, Berend et al. [3] did report higher average Harris hip scores and Lower Extremity Activity Scale scores to a level of statistical significance in a series of 605 patients comparing the DAA to the direct lateral approach. In a retrospective comparative study between the DAA and the posterior approach at 3 weeks post-operatively, Nakata et al. [1] concluded the DAA resulted in a more rapid recovery of hip function and gait ability. They compared measures of single-leg stance of more than 5 seconds, differences in 50 meter walking time and duration of time requiring a walking aid to make these conclusions. Lugade et al. found a statistically significant improvement in gait symmetry at 6 weeks comparing the DAA to the anterolateral approach [18].

Berend et al. [3] found no difference in the length of hospital stay between the DAA and the direct lateral approach, but reported an increased number of DAA patients discharged directly to home instead of an extended care facility. Although the average patient age of each group was 63 years, the ages of the subgroups of patients discharged to home compared to an extended care facility was not provided, thus younger patients who were more suited for a discharge to home could account for the difference if in fact there was a difference in the mean age of these two groups. Goebel et al. [19] reported a shorter hospital stay to a level of statistical significance with a DAA group compared to a transgluteal group, however the difference was 10 days vs. 13 days in a German hospital. 
We found that patients perceived less acute pain after surgery and throughout the hospitalization period. These findings are partially in agreement with Goebel et al. [19] who reported a statistically significant decrease in acute post-operative pain medication on the first day after surgery following primary total hip arthroplasty through the direct anterior approach compared to the transgluteal lateral approach in a retrospective cohort study including 200 patients, but no difference in the amount of pain medication consumed for the remaining period of hospitalization.

We are not aware of any studies in the literature that compare time to return to work after total hip replacement between the DAA to other surgical approaches. In our study group patients reported returning to work sooner with the DAA approach; however our small sample size of patients surveyed who planned to return to work after surgery makes it difficult to draw conclusions with respect to how the surgical approach affects how rapidly patients can return to work which is also occupation dependent.

The results of the questionnaire do not appear to be affected by multi-modal pain management, but we acknowledge without directly comparing the route of administration, type and amount of pain medication utilized in the post-operative period, it is impossible to conclude that multi-modal pain management did not have some influence on patient responses.

Patient's overall been more satisfied and preferred the postoperative recovery following the direct anterior approach. However, patients less than 70 years of age were more likely to have their preoperative expectations fulfilled with the direct anterior approach than a traditional approach.

We acknowledge a prospective randomized study is the only way to definitively draw conclusions about the effectiveness of one surgical approach over another on patient's perception of whether or not a real difference exists. Our data suggests that such a study that is sufficiently powered to detect a real clinical difference if one exists is worth pursuing. We also acknowledge our results are subject to selection and recall bias. With the large amount of information accessible to patients, they could have specifically selected a surgeon who utilizes the direct anterior approach for total hip replacement. With an average time of seven years between each hip replacement, there is certainly potential for recall bias to influence patient's responses. The direct anterior approach was the second operation for all hip replacements and having been through the experience once before, patients already had an idea about what to anticipate after the second surgery which could have also biased the results.

It is important to note that the patients in this study received their total hip arthroplasties with the DAA before this approach was heavily marketed. Thus, patients were not self-selected to expect a quicker recovery, and we did not appreciate patient expectations as a possible source of bias.

In conclusion, patients perceive less acute pain, a faster surgical recovery, and improved post-operative function with their hip replacement through the DAA compared to a traditional approach. Long-term measures of pain relief function or strength did not differ between approaches. However, patients less than 70 years of age are more likely to report a preference for the DAA and perceived the hip replaced through the DAA to be associated with a faster surgical recovery, pre-operative expectations were more closely met, and they were more likely to recommend the results of their hip replaced using the DAA approach over their other hip replacement to a friend or loved one.

\section{Acknowledgements}

The authors are grateful to Linda Franklin, $\mathrm{RN}$ for all her time and hardwork with data collection for this study.

\section{Disclosures}

Dr. Moskal is a paid consultant and has an ongoing relationship with Depuy Orthopedics. Dr. Scanelli and Dr. Capps have no relevant financial conflicts of interest to disclose.

\section{References}

1. Nakata K, Nishikawa M, Yamamoto K, Hirota S (2009) A Clinical Comparative Study of the Direct Anterior With Mini-Posterior Approach. The Journal of Arthroplasty 24: 698

2. Restrepo C,Parvizi J, Pour AE, Hozack WJ (2010) Prospective Randomized Study of Two Surgical Approaches for Total Hip Arthroplasty. The Journal of Arthroplasty 25: 671

3. Berend KR, Lombardi AV Jr, Seng BE, Adams JB (2009) Enhanced early outcomes with the anterior supine intermuscular approach in primary total hip arthroplasty. J Bone Joint Surg Am 91 Suppl 6: 107-120.

4. Anterior Total Hip Arthroplasty Collaborative Investigators, Bhandari M, Matta JM, Dodgin D, Clark C, et al. (2009) Outcomes following the single-incision anterior approach to total hip arthroplasty: a multicenter observational study. Orthop Clin North Am 40: 329-342.

5. Siguier T, Siguier M, Brumpt B (2004) Mini-incision anterior approach does not increase dislocation rate: a study of 1037 total hip replacements. Clin Orthop Relat Res : 164-173.

6. Restrepo C, Mortazavi SM, Brothers J, Parvizi J, Rothman RH (2011) Hip dislocation: are hip precautions necessary in anterior approaches? Clin Orthop Relat Res 469: 417-422.

7. Matta JM, Shahrdar C, Ferguson T (2005) Single-incision anterior approach for total hip arthroplasty on an orthopaedic table. Clin Orthop Relat Res 441 115-124.

8. Bergin PF, Doppelt JD, Kephart CJ, Benke MT, (2010) Comparison of Minimally Invasive Direct Anterior Versus Posterior Total Hip Arthroplasty Based on Inflammation and Muscle Damage Markers. The Journal of Bone \& Joint Surgery: 1392

9. Meneghini RM, Pagnano MW, Trousdale RT, Hozack WJ (2006) Muscle damage during MIS total hip arthroplasty: Smith-Petersen versus posterior approach. Clin Orthop Relat Res 453: 293-298.

10. Bremer AK, Kalberer F, Pfirmann CW, Dora C (2011) Soft-tissue changes in hip abductor muscles and tendons after total hip replacement: comparison between the direct anterior and the transgluteal approaches. J Bone Joint Surg $\mathrm{Br}$ 93: 886-889.

11. Mancuso CA, Jout J, Salvati EA, Sculco TP (2009) Fulfillment of patients' expectations for total hip arthroplasty. J Bone Joint Surg Am 91: 2073-2078.

12. Mancuso CA, Salvati EA, Johanson NA, Peterson MG, Charlson ME (1997) Patients' expectations and satisfaction with total hip arthroplasty. J Arthroplasty 12: 387-396.

13. Callaghan JJ, Albright JC, Goetz DD, Olejniczak JP, Johnston RC (2000) Charnley total hip arthroplasty with cement. Minimum twenty-five-year followup. J Bone Joint Surg Am 82: 487-497.

14. Nilsdotter AK, Petersson IF, Roos EM, Lohmander LS (2003) Predictors of patient relevant outcome after total hip replacement for osteoarthritis: a prospective study. Ann Rheum Dis 62: 923-930.

15. Klausmeier V, Lugade V, Jewett BA, Collis DK, Chou LS (2010) Is there faster recovery with an anterior or anterolateral THA? A pilot study. ClinOrthopRelat Res 468: 533-541.

16. Maffiuletti NA, Impellizzeri FM, Widler K, Bizzini M, Kain MS, et al. (2009) Spatiotemporal parameters of gait after total hip replacement: anterior versus posterior approach. Orthop Clin North Am 40: 407-415.

17. Mayr E, Nogler M, Benedetti M-G, Kessler O, Reinthaler A, et al. (2009) A prospective randomized assessment of earlier functional recovery in THA patients treated by minimally invasive direct anterior approach: A gait analysis study. Clinical Biomechanics 24: 812 . 
Citation: Moskal JT, Scanelli JA, Capps SG (2014) Patient Satisfaction after Total Hip Arthroplasty Comparing the Direct Anterior Approach with other Standard Approaches. Surgery Curr Res 4: 174. doi:10.4172/2161-1076.1000174

18. Lugade V, Wu A, Jewett B, Collis D, Chou LS (2010) Gait asymmetry following an anterior and anterolateral approach to total hip arthroplasty. ClinBiomech (Bristol, Avon) 25: 675-680
19. Goebel S, Steinert AF, Schillinger J, Eulert J, Broscheit J, et al. (2012) Reduced postoperative pain in total hip arthroplasty after minimal-invasive anterior approach. Int Orthop 36: 491-498. 